Original ReseArCh ARTICLE

\title{
Diagnosis and Treatment of 12 Cases of Traumatic Duodenal Rupture
}

\author{
Jianzhen Du,Dongying He,Jiacun Wang \\ Meizhou Medical College, Guangdong, China
}

\begin{abstract}
Due to the frequent occurrence of modern traffic accidents and accidental injuries, duodenal rupture is also common. As the duodenal anatomical location deep, preoperative diagnosis of duodenal rupture is more difficult, once

misdiagnosed, the consequences of serious

KEYWORDS: traumatic; duodenal rupture; diagnosis and treatment
\end{abstract}

Due to the frequent occurrence of modern traffic accidents and accidental injuries, duodenal rupture is also common. Due to the depth of the duodenal anatomy, preoperative diagnosis of duodenal rupture is more difficult, once misdiagnosed, the consequences are serious. Our hospital from January 2004 to June 2006 treated traumatic duodenal rupture in 12 cases, timely diagnosis, timely treatment, the results were satisfactory. The report is as follows.

\section{Clinical data}

General information in this group of 12 cases, including 8 males and 4 females, aged 20 to 53 years (mean 35 years); 9 cases of accidents, 2 cases of falling injuries, knife stab in 1 case. After treatment, the average time of $4 \mathrm{~h} .12$ cases had upper abdominal or right upper quadrant pain, localized peritonitis, of which 4 cases of preoperative shock. Abdomen in 5 cases out of non-coagulation, color Doppler ultrasound examination in 8 cases were peritoneal effusion and retroperitoneal hematoma, CT examination in 6 cases were peritoneal effusion and retroperitoneal hematoma (2 cases showed retroperitoneal and right kidney gap with air bubbles, oral Diatrizoate in 4 cases with spillover). 5 cases were diagnosed preoperative and 7 cases were diagnosed intraoperative. Surgery was found in 1 case of duodenal rupture, 9 cases of descending, 2 cases of horizontal, combined with liver and spleen rupture in 5 cases, 1 case of pancreatic injury, duodenal rupture, 4 cases of intestinal perimeter, $<1 / 3 \sim 1 / 2$ weeks in 6 cases, $>1 / 2$ weeks in 2 cases.

Surgical approach to deal with intra-abdominal active bleeding. Simple duodenal rupture repair + omentum covered in 4 cases, simplified duodenal diverticulum in 8 cases. Surgery were placed in the rupture of the mouth of the distal end of the tube, the hole of the hole, the right diaphragm, pelvic catheter were drainage.

\section{Results}

11 cases were discharged and 1 case died due to multiple organ failure. Follow-up of 1-3 years, 3 cases with postoperative intestinal adhesion by symptomatic treatment improved, more than no discomfort.

\section{Discussion}

Timely diagnosis of early surgery is the key to successful treatment of duodenal rupture occurred in the second and third duodenum, because of its unique anatomical relationship and physiological characteristics, with the stomach, gallbladder, pancreas are closely related, Symptoms and signs are not typical, and easily adjacent to the organ or systemic complications of the clinical manifestations of the cover, easily lead to delayed diagnosis and treatment, and even during surgery, the doctor on the lack of understanding of upper abdominal hematoma, failed to explore the duodenum Missed, especially retroperitoneal rupture to be more attention to clinicians. Duodenal rupture preoperative diagnosis is more difficult, especially early diagnosis of retroperitoneal rupture is more difficult. Combined with the actual group, to do early diagnosis and timely detection of retroperitoneal duodenal rupture, should pay attention to the following points: (1) where severe abdominal injury should be thought of duodenal rupture may be. If suspected duodenal rupture, should be closely observed, repeated repeated upper abdominal examination, the use of a variety of 
means of joint examination and abdominal puncture, if necessary, timely laparotomy; (2) the correct use of laboratory and auxiliary examination. Amylase increased a certain significance, especially when combined with pancreatic injury; abdominal puncture, such as withdrawal of bloody or bile-like fluid should be considered duodenal rupture may be; $\mathrm{X}$-ray examination can be seen right kidney and right diaphragm around the foot of the free gas, The upper right lumbar muscle and kidney shadow blurred; B ultrasound, CT visible right upper abdomen local effusion or qi gas [1]; oral diatrizoate angiography visible duodenum contusion agent; (3) laparotomy Should be fully aware of the meaning of right upper and lower retroperitoneal hematoma. If the surgery found retroperitoneal hematoma, free gas and bile staining, should consider retroperitoneal duodenal rupture [1].

Select a reasonable surgical current duodenal rupture of the surgical methods are the following: (1) simple suture repair; (2) jejunum or stomach patch repair; (3) duodenal jejunum Roux-Y Anastomosis; (4) duodenal diverticulum surgery and simplified surgery; (5) duodenostomy decompression surgery; (6) pancreaticoduodenectomy. The authors believe that patients with retroperitoneal duodenal rupture most of the merger with severe organ damage, vital signs of instability, surgery should be simple and effective. In recent years, undergraduate use similar to the pancreas bundling technique [3], duodenal diverticulitis surgery and simplified surgery to simplify, to achieve satisfactory results, specific methods: (1) duodenal rupture debridement suture repair, plus (3) gastric jejunum anastomosis; (4) porous gastric tube into the duodenum rupture mouth below; (2) with the absorption of a needle tied to the stomach pylorus, the stomach content temporarily turn; 5) duodenal rupture repair treatment of double casing 1 root. The procedure is relatively simple, and play the role of duodenal diverticulitis, surgery time is short, trauma, drainage smooth, duodenal emptying favorable rupture healing, 1 to 3 months after the pylorus suture absorption, the stomach duodenum can be recanalized.

Perioperative treatment of duodenal rupture patients have insufficient blood volume, acidosis and shock, postoperative prone to renal dysfunction, multiple organ failure, abdominal infection and pulmonary infection, so perioperative treatment is The key to successful surgery. Preoperative and postoperative anti-shock and anti-infection treatment, to maintain drainage after the patency, to correct electrolyte imbalance, to maintain water and electrolyte, acid-base balance and nutritional support treatment (TPN), to be stable recovery of intestinal function After the tube to restore the diet $[4,5]$.

\section{$\underline{\text { References }}$}

1. CT findings of duodenal injury (report of 12 cases). Zhejiang Journal of Clinical Medicine, 2007,9 (1): 123.

2. He Sanguang, Xia Zhiping, Tian Liguo, editor. Chinese surgical expert experience collection. 2nd edition. Shenyang: Shenyang Publishing House, $2000.573 \sim 575$.

3. Peng Shuyue, Peng Chenghong. Bundled pancreatic anastomosis (report of 28 cases). Chinese Journal of Surgery, 1997,51 (3): 158.

4. Wu Jieping, Qiu Fazu, editor. Huang Jia Si surgery. 6th edition. Beijing: People's Health Publishing House, 2000.960.

5. Wu Zaode, Wu Zhaoxhan, editor. Surgery. 6th edition. Beijing: People's Health Publishing House, $2003.428 \sim 429$. 